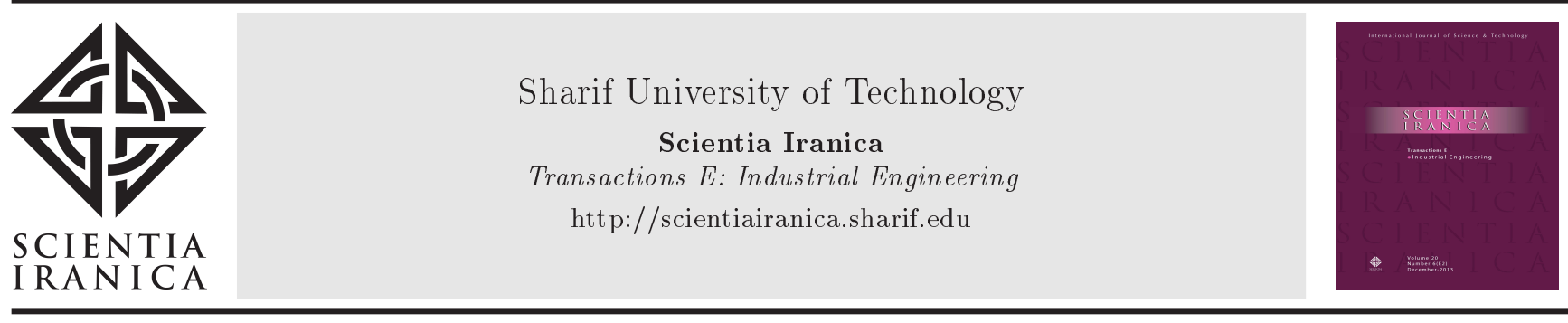

\title{
Optimal fleet composition and mix periodic location-routing problem with time windows in an offshore oil and gas industry: A case study of National Iranian Oil Company
}

\author{
M. Amiri ${ }^{a}$, S.J. Sadjadi ${ }^{\text {b,* }}$, R. Tavakkoli-Moghaddamc, and A. Jabbarzadeh ${ }^{\text {b }}$ \\ a. Department of Industrial Engineering, Science and Research Branch, Islamic Azad University, Tehran, Iran. \\ b. School of Industrial Engineering, Iran University of Science and Technology, Tehran, Iran. \\ c. School of Industrial Engineering, College of Engineering, University of Tehran, Tehran, Iran.
}

Received 3 March 2017; received in revised form 13 September 2017; accepted 18 December 2017

\section{KEYWORDS \\ Maritime \\ transportation; \\ Supply vessel; \\ Fleet composition; \\ Location-routing problem.}

\begin{abstract}
This paper presents a new Mixed-Integer Non-Linear Programming (MINLP) model for a Supply Vessel Planning (SVP) problem. The traditional SVP, which is a maritime transportation problem, is developed into a Maritime Fleet Sizing Mix Periodic Location-Routing Problem with Time Windows (MFSMPLRPTW) by considering suppliers, location of onshore base(s), and some real-life aspects. The objective of this model is to decide the composition of fleets, optimal voyages, schedules, and the optimal location(s) for onshore base(s) in such a way that the total cost is minimized and the needs of operation regions are fulfilled. The MFSMPLPRTW model is solved by an exact twophase solution approach for both small and medium cases. Moreover, two meta-heuristic algorithms are used to solve the large-sized instances. In order to justify and show how the model and solution can lead to significant economic improvements for real-life instances, a case study by the IOOC is considered, which is the only offshore oil and gas producer in Iran that has lots of installations and operation regions in the Persian Gulf and the Sea of Oman.
\end{abstract}

(C) 2019 Sharif University of Technology. All rights reserved.

\section{Introduction}

Upstream is one of the most important parts of an offshore oil and gas supply chain that depends on maritime transportation in order to produce oil and gas from offshore installations and operation regions. Figure 1 shows an upstream oil and gas logistics [1]. All oil and gas offshore installations and operation regions require materials, equipment, and other consumables

\footnotetext{
*. Corresponding author.

E-mail address: sjsadjadi@iust.ac.ir (S.J. Sadjadi).
}

from onshore base(s) and they have to return some backloads to land regularly by supply vessels to ensure efficient and continuous production. Since the rate of chartering of supply vessels is very expensive to minimize the transportation costs, the optimal number and type of supply vessels and reduction of sailing distances should be considered [2]. In the following, the studies on the Maritime Fleet Size and Mix Problems (MFSMP) as a strategic and tactical problem are presented [3].

The strategic level contains the decisions for longterm planning. A wide spectrum consisting of designing the transportation services and accepting longterm contracts exists at the maritime transportation 


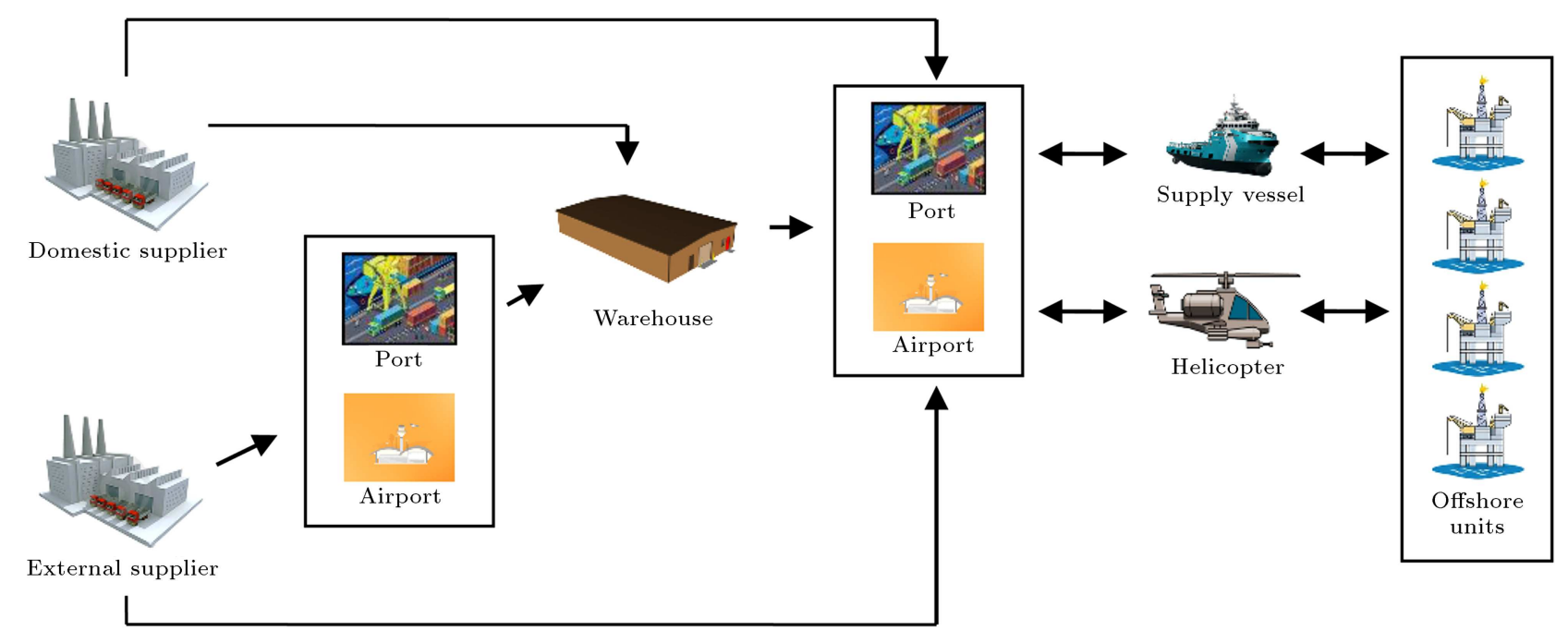

Figure 1. Upstream offshore oil and gas logistics.

strategic level. On the other hand, at the tactical planning level, the focus is on medium-term decisions, and the most important decisions at this level in maritime transportation are associated with routing and scheduling decisions [4].

Everett et al. [5] presented the first study on the long-term MFSMP in 1972. A Linear Programming (LP) was used to determine the number of needed ships for the US foreign trade. Murotsu and Taguchi [6] introduced a new model in 1975 by combining Dynamic Programming (DP) with Non-Linear Programming (NLP) to obtain the optimal size of a fleet of oil carriers. Larson [7] studied transferring sludge from the city of New York to an offshore site to obtain the number and the size of needed supply vessels in 1988. In another study, Pesenti [8] presented a heuristic method to purchase container ships in 1995. In order to find the possibility of building different ships, Sigurd et al. [9] presented a new model solved by a Branchand-Price (B\&P) algorithm. Zeng and Yang [10] introduced a new model of the problem by considering the fleet size and mix as well as ship schedules for a coal shipping system in China. They used a Tabu Search (TS) algorithm, which was presented by Glover and Laguna [11] in 1997, to solve the resulted model. Fagerholt et al. [12] in 2010 presented a new method to evaluate different alternatives of fleets by using simulation tools.

For the first time, a fleet size and mix Vehicle Routing Problem (VRP) model [13] was presented by Kocü et al. [14] by considering heterogeneous fleet and time windows. Next, Fagerholt and Lindstad [15] presented a real short-term problem of maritime transportation in the Norwegian Sea. Aas et al. [16] considered a real-life routing problem in the Norwegian oil and gas industry, which showed how the limitations of offshore installation's capacity affected the optimal routes. Gribkovskaia et al. [17] discussed a routing problem by considering delivery, pick up, backloads, and the limitation of offshore installation's capacity. Iachan [18] described a fleet composition and routing problem for a special kind of vessels in Petrobras, the biggest Brazilian oil company. They used a Genetic Algorithm (GA) proposed by Holland [11] in 1975 to solve their model. Aas et al. [19] discussed the role of supply vessels as the largest cost element in upstream logistics. They focused on designing capacity, loading, and unloading capabilities and sailing as the most important features of supply vessels that must be noticed.

Shyshou et al. [20] presented a fleet sizing problem for anchor handling operations in offshore mobile installations. A simulation method was presented to determine the cost-optimal fleet of vessels for a longterm hire to handle future operations. HalvorsenWeare and Fagerholt [21] discussed several approaches in order to create a robust supply vessel planning problem. In their model, the weather condition was considered as an important element of the supply vessels scheduling. Halvorsen-Weare et al. [2] presented a two-stage voyage-based approach in order to find the optimal fleet composition and routing for some offshore installations, which must be supplied with some consumables in the Norwegian Sea for Statoil Company. In another study, Shyshou et al. [22] presented a heuristic method for periodic supply vessel planning, which was capable of solving large-sized instances.

Norlund et al. [23] focused on speed of supply vessels as an important element in order to reduce emissions and routing costs of supply vessels. The results showed robustness improvements and increased emissions and costs together. Christiansen et al. [24] considered an arc-flow and a path-flow model for a fleet of fuel supply vessels in order to serve the ships 
anchored in a port. Cuesta et al. [1] presented an extension of the Vessel Routing Problem by considering Selective Pickups and Deliveries (VRPSPD) in an offshore oil and gas industry with not many changes in current planning. They also presented a Multi Vessel Routing Problem with Pickups and Deliveries (MVRPPD) model with more economic improvements, which required more changes in current planning.

The optimal fleet composition and designing routes and also the locations of the onshore base(s) are very critical for oil and gas companies [14]. Studies have shown that the location and routing decisions are inter-dependent and the total cost increases if they are considered separately. The Location-Routing Problems (LRPs) include a set of potential depots with opening costs and a set of customers with known demands supplied by designing vehicle routes in such a way that the total cost is minimized [25]. The classical LRPs have been studied significantly during the last decades. After the survey by Nagy and Salhi [26], the classical LRPs, by considering the capacity of depots and capacity of vehicles, were named Capacitated LRPs (CLRPs). The LRPs are NP-hard combinatorial optimization problems and lots of algorithms have been introduced to solve them, similar to Greedy Randomized Adaptive Search (GRASP) by Prins et al. [27], GA by Derbel et al. [28], Simulated Annealing (SA) by Yu et al. [29], Adaptive Large Neighborhood Search (ALNS) by Hemmelmayr et al. [30], and Variable Neighborhood Search (VNS) by Jarboui et al. [31]. In addition, there are some studies that use exact methods such as Laporte and Nobert (branch-andbound algorithm) [32], Albareda-Sambola et al. (lower bound) [33], and Belenguer et al. (Branch-and-Cut algorithm) [34].

A Maritime Fleet Sizing Mix Periodic LocationRouting Problem with Time Windows (MFSMPLRPTW) model having real-life aspects is presented in this paper. The purpose of the MFSMPLRPTW model is to minimize the total costs of chartering heterogeneous supply vessels, opening costs of onshore base(s), sailing costs, transportation costs, and selected suppliers, while all customers' demands are met at a right time.

The model is solved by using GAMS software (CPLEX solver) for small-sized instances. A Particle Swarm Optimization (PSO) algorithm [35], as one of the most popular swarm-based algorithms, and a Whale Optimization Algorithm (WOA) [36] are used to solve large-sized instances in a reasonable amount of time. The PSO has been widely used to solve VRPs, location problems, scheduling and fleet sizing problems by researchers, such as Marinakis and Marinaki [37], Belmecheri et al. [38], and Onwunalu and Durlofsky [39].

The WOA is a new swarm-based meta-heuristic algorithm, which was presented in 2016. It imitates the way of humpback whales to search for food. In the first phase of the WOA (i.e., exploitation phase), the way of swimming and moving around prey and creating special bubbles in a circle like ' 9 '-shaped by humpback whales are presented. In the second phase of WOA (i.e., exploration phase), whales try to find a prey randomly by humpback whales [36]. Many researchers in different areas have been motivated to use the WOA because of some important features, such as adjusting just two parameters, easy implementation, and searching the border areas by using the logarithmic spiral function.

Kaveh and Ghazaan [40] presented an Enhanced Whale Optimization Algorithm (EWOA) in order to improve the solution accuracy, reliability, and convergence speed of the sizing optimization problems. Mafarja and Mirjalili [41] presented a hybrid WOA with SA for feature selection. In another study carried out by Prakash and Lakshminarayana [42], the WOA was used to find the optimal siting and placement of electrical equipment for a typical radial distribution system. Reddy et al. [43] used the WOA to decide on the optimal number and placement of distributed generators. In another study carried out by $\mathrm{Abd} \mathrm{El}$ Aziz et al. [44], the WOA was used to determine the optimal multi-level threshold for image segmentation. The experimental results showed that the proposed algorithm outperformed the other swarm algorithms.

The contributions of this paper are as follows. The MFSMPLRPTW model is presented as a new supply vessel planning problem by considering some potential onshore base(s) with different features. Different suppliers with various costs, such as capacities and transportation costs, are discussed for the first time in this paper. In addition, some novel real-life aspects (e.g., installing central warehouse(s) at onshore base(s) and the limitation of base's capacity to service supply vessels) are considered.

The reminder of this paper is organized as follows. The problem definition is presented in Section 2. Then, the mathematical model is discussed in Section 3. After that the solution methodology is presented in Section 4, and the computational results are shown in Section 5. The performance of meta-heuristic algorithms is discussed in Section 6 and finally the conclusion is stated in Section 7.

\section{Problem definition}

In this section, at first, the traditional SVP problems are described; then, the new model (FSMPLRPTW) is introduced by considering the optimal location of onshore base(s) to install central warehouse(s) in offshore oil and gas industry. The traditional SVP problem is a mixture of fleet composition problem and Periodic Vehicle Routing Problem (PVRP) that some offshore 

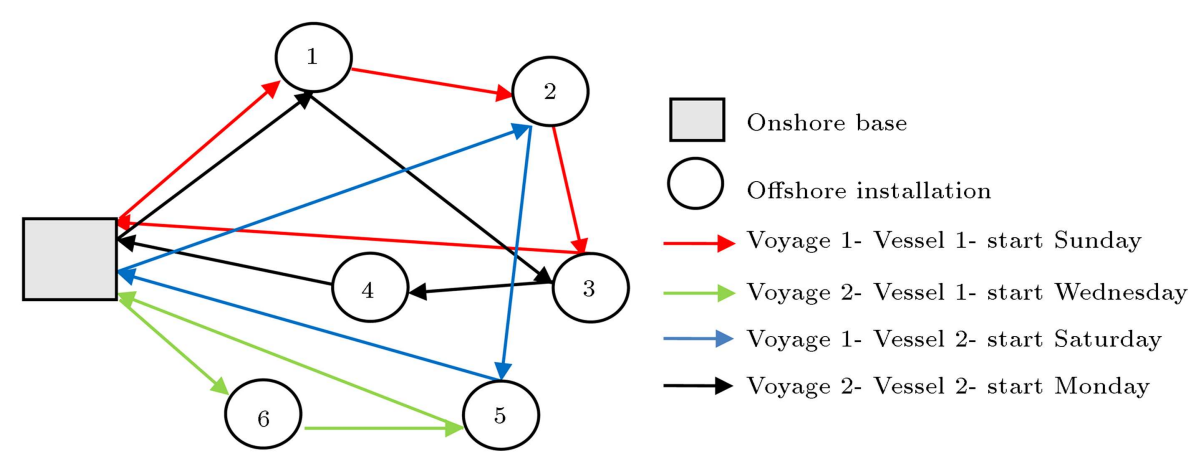

Figure 2. Traditional SVP with one onshore base.

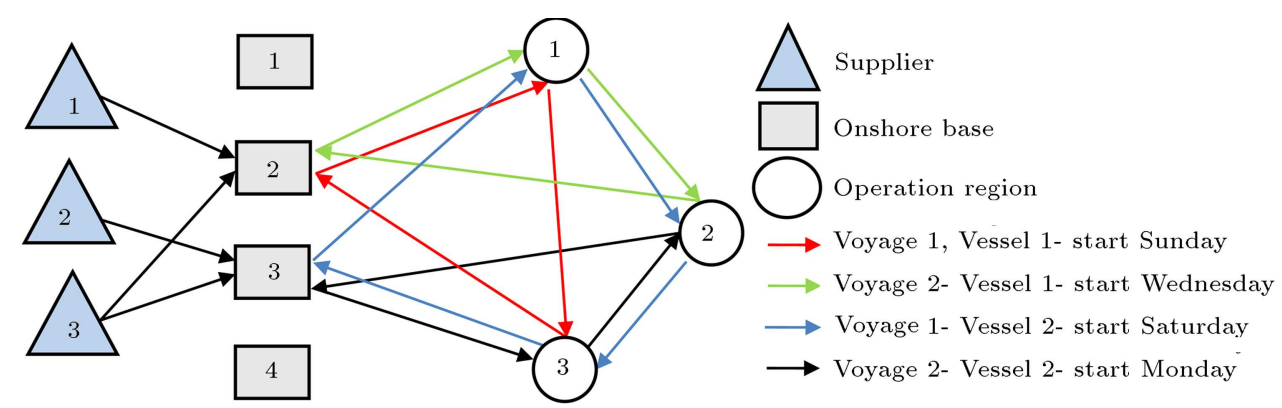

Figure 3. New model of SVP with three suppliers and four potential onshore base(s).

installations with certain demands should be supplied by supply vessels. The planning period is up to one week and supply vessels start and end their voyages at one common port during the week. Each voyage may visit one or more installations during the week, as depicted in Figure 2. The objective of this model is to identify the optimal composition of fleets and also voyages and schedules at the same time, while the total cost should be minimized and the needs of offshore installations must be supplied reliably [2].

The objective of the new model is to locate optimal onshore base(s) to select the best suppliers and to identify the best voyages and optimal fleet composition at the same time by considering the minimum cost and sending cargoes reliably. The related problem is illustrated in Figure 3. It is supposed that the central warehouse(s) is installed at the optimal onshore base(s). In the following, the special constraints of the problem in italic and are presented.

Onshore base and operation regions may have opening hours (Time Windows), where loading and unloading are only permitted at this time. The opening hours are set between 08:00 and 16:00 for potential onshore base(s) and between 07:00 and 19:00 for operation regions. The service time required for loading at onshore base(s) has been estimated as about eight hours; therefore, each supply vessel should be ready at the onshore base before 08:00 in order to load cargoes and start the voyage at 16:00.

The demand of operation regions is estimated for one week (Periodic). In addition, some operation regions need to be visited in a number of certain times by supply vessels during the week. By using this limitation, we always have to use extra supply vessels, which can have extra sailing costs or extra renting costs.

The capacities of suppliers, potential onshore base(s), and operation regions are limited. Different suppliers have various capacities and transportation costs to send cargoes, and it is supposed that all needed cargoes are sent directly to onshore base(s) and are available in optimal onshore base(s) at the beginning of the period. Moreover, the capacity limitation on the number of supply vessels to berth in potential onshore base is considered for the first time. Central warehouse(s) is planned to be installed in optimal onshore base(s). On the other hand, the capacity of operation regions during a week varies, and specific volume of cargoes can be unloaded on a certain day.

Experiences have shown that the deck capacity of supply vessels is more important than the bulk capacity to send cargoes. Thus, the demands of operation regions are considered in a cubic meter. All demands of operation regions on a certain day and on a certain voyage must not exceed the capacity of the supply vessel, which has been selected to carry them. It is supposed that the volume of back loads of operation regions is less than the demands; thus, there are enough spaces to carry them.

In the offshore oil and gas industry, spread the 
departures is necessary; otherwise, the total costs increase. For example, if an operation region needs two visits during a week and are made during the first two days of that week and there is a necessary visit to this operation region after Tuesday, another supply vessel or a helicopter has to be sent, which is very costly in most cases.

Too short voyages (less than 2 days) with very few visits (less than 2 visits) because of non-optimal usage of supply vessel's capacity and too long voyages (more than 2 days) with many visits (more than 5 visits) because of the uncertainty are not allowed [45].

\section{Mathematical model}

This section presents the mathematical model for the MFSMPLRPTW problem. The objective function shown by $Z$ is to select the most cost-effective supply vessels and suppliers and also to locate optimal onshore base(s) to berth supply vessels and pick the best generated voyages, which fulfill the constraints. Table 1 presents the notations used in the model.

It is supposed that all the routes are generated at first and considered as a set in the mathematical model. Generating routes is done through phase 1 of the methodology presented by Halvorsen-Weare et al. [2] and will be introduced in the next section. The mathematical model is presented below:

$$
\begin{aligned}
\min Z= & \sum_{k \in V} c_{k}^{c h} Z_{j k} \\
& +\sum_{j \in B} c_{j}^{b f} Y_{j}+\sum_{s \in S} \sum_{j \in B} c_{s j}^{s b} w_{s} Q_{s j}^{s} \\
& +\sum_{j \in B} \sum_{k \in V} \sum_{r \in R} \sum_{t \in T} c_{j k r}^{s c} X_{j k r t} \\
& +\sum_{j \in B} \sum_{i \in N} \sum_{k \in V} \sum_{r \in R} \sum_{t \in T} c_{j}^{b v} Q_{j i k r t}^{b},
\end{aligned}
$$

s.t.

$$
\begin{aligned}
& \sum_{j \in B} \sum_{k \in V} \sum_{r \in R} \sum_{t \in T} a_{i r} X_{j k r t} Q_{j i k r t}^{b}-m_{i}=0, \quad i \in N \\
& \sum_{j \in B} \sum_{k \in V} \sum_{r \in R} \sum_{t \in T} a_{i r} X_{j k r t} Z_{j k}-s n_{i} \geq 0, \quad i \in N \\
& \sum_{j \in B} \sum_{k \in V} \sum_{r \in R} Q_{j i k r t}^{b}-q_{i t}^{c} \leq 0, \quad i \in N, t \in T \\
& \sum_{j \in B} Q_{s j}^{s}-c a p_{s}^{s u} \leq 0, \quad s \in S \\
& \sum_{s \in S} Q_{s j}^{s}-\left(c a p_{j}^{b} Y_{j}\right) \leq 0, \quad j \in B
\end{aligned}
$$

$$
\begin{aligned}
& \sum_{s \in S} Q_{s j}^{s}-\sum_{i \in N} \sum_{k \in V} \sum_{r \in R} \sum_{t \in T} Q_{j i k r t}^{b}, \quad j \in B, \\
& \sum_{j \in B} Y_{j}-p \leq 0, \\
& \sum_{j \in B} Z_{j k} Y_{j}-1 \leq 0, \quad k \in V, \\
& \sum_{k \in V} \sum_{r \in R} X_{j k r t}-u_{j t} \leq 0, \quad j \in B, t \in T, \\
& \sum_{j \in B} \sum_{r \in R} \sum_{t \in T} t i_{j k r} X_{j k r t}-f_{k} \sum_{j \in B} Z_{j k} \leq 0, \\
& t i_{j k r} X_{j k r t}-l r \geq 0, \quad j \in B \in V, k \in V, r \in R, t \in T, \\
& t i_{j k r} X_{j k r t}-h r \leq 0, \quad j \in B, k \in V, r \in R, t \in T,
\end{aligned}
$$$$
\sum_{r \in R} X_{j k r t}+\sum_{r \in R} \sum_{g=1}^{l-1} X_{j k r((t+g) \bmod |T|)}-1 \leq 0,
$$

$$
j \in B, k \in V, t \in T, l \in L,
$$

$\sum_{i \in N} Q_{j i k r t}^{b}-\operatorname{cap}_{k}^{v} \leq 0, \quad j \in B, k \in V, r \in R, t \in T$,

$\sum_{j \in B} \sum_{k \in V} \sum_{r \in R} a_{i r} X_{j k r t}-1 \leq 0, \quad i \in N, t \in T$,

$\sum_{j \in B} \sum_{k \in V} \sum_{r \in R} \sum_{g=0}^{2} a_{i r} X_{j k r((t+g) \bmod |T|)}-1 \leq 0$,

$$
i \in N_{2}, t \in T
$$

$\sum_{j \in B} \sum_{k \in V} \sum_{r \in R} \sum_{g=0}^{2} a_{i r} X_{j k r((t+g) \bmod |T|)}-1 \geq 0$,

$$
i \in N_{3}, t \in T
$$

$\sum_{j \in B} \sum_{k \in V} \sum_{r \in R} \sum_{g=0}^{2} a_{i r} X_{j k r((t+g) \bmod |T|)}-2 \geq 0$,

$$
i \in N_{4}, t \in T
$$

$Z_{j k} \in[0,1], \quad j \in B, k \in V$,

$Y_{j} \in[0,1], \quad j \in B$,

$X_{j k r t} \in[0,1], \quad j \in B, k \in V, r \in R, t \in T$,

$Q_{j i k r t}^{b} \geq 0, \quad j \in B, i \in N, k \in V, r \in R, t \in T$, 
Table 1. Definition of sets, indexes, parameters, and variables.

\begin{tabular}{|c|c|}
\hline Sets & Definition \\
\hline$N$ & The set of all offshore operation regions \\
\hline$B$ & The set of alternative onshore base(s) \\
\hline$V$ & The set of supply vessels \\
\hline$R$ & All generated voyages \\
\hline$T$ & The set of days in the planning horizon \\
\hline$S$ & The set of suppliers \\
\hline$L$ & The set of allowable duration of supply vessels \\
\hline$N_{2}$ & The operation regions that need two visits in the planning horizon \\
\hline$N_{3}$ & The operation regions that need three visits in the planning horizon \\
\hline$N_{4}$ & The operation regions that need four visits in the planning horizon \\
\hline Indexes & Definition \\
\hline$i$ & Operation regions index \\
\hline$j$ & Onshore-base(s) index \\
\hline$k$ & Supply vessels index \\
\hline$r$ & Routes index \\
\hline$t$ & Days index \\
\hline Parameters & Definition \\
\hline$C_{k}^{c h}$ & The weekly cost per unit chartered and used supply vessel $k$ per period \\
\hline$C_{j k r}^{S C}$ & The sailing cost of supply vessel $k$ from onshore base $j$ on route $r$ \\
\hline$C_{j}^{b f}$ & The cost of installing the central warehouse(s) at onshore base $j$ \\
\hline$C_{j}^{b v}$ & The variable cost per unit of cargo at onshore base $j$ \\
\hline$C_{s j}^{s b}$ & The transportation cost from supplier $s$ to onshore base $j$ \\
\hline$t i_{j k r}$ & The duration of voyage $r$ sails by supply vessel $k$ from onshore base $j$ \\
\hline$f_{k}$ & The allowable days of sailing supply vessel $k$ \\
\hline$u_{t j}$ & The number of allowable supply vessels at onshore base $j$ \\
\hline$m_{i}$ & The demand of operation region $i$ \\
\hline$s n_{i}$ & The number of needed visits to operation region $i$ \\
\hline$a_{\text {ir }}$ & Representing if operation region $i$ is visited on route $r$ or not \\
\hline$c a p_{k}^{v}$ & The capacity limitation of supply vessel $k$ \\
\hline$c a p_{j}^{b}$ & The capacity limitation of onshore base $j$ \\
\hline $\operatorname{cap}_{s}^{s u}$ & The capacity limitation of supplier $s$ \\
\hline $\operatorname{lr}$ & The minimum allowable duration of voyages \\
\hline$h r$ & The maximum allowable duration of voyages \\
\hline$q_{i t}^{c}$ & The capacity limitation for unloading of operation region $i$ \\
\hline$w_{s}$ & The weight factors for supplier $s$ \\
\hline$p$ & The number of needed onshore base(s) \\
\hline Variables & Definition \\
\hline$Z_{j k}$ & One if supply vessel $k$ is assigned to onshore base $j ; 0$ otherwise \\
\hline$Y_{j}$ & One if onshore base $j$ is selected to berth supply vessel $k ; 0$ otherwise \\
\hline$X_{j k t}$ & One if supply vessel $k$, on route $r$, from onshore base $j$ and on day $t$ sails; 0 otherwise \\
\hline$Q_{j i k r t}^{b}$ & The quantity of sending cargoes from onshore base $j$ to operation region $i$ \\
\hline$Q_{s j}^{s}$ & The quantity of sending cargoes from supplier $s$ to onshore-base $j$ \\
\hline
\end{tabular}




$$
Q_{s j}^{s} \geq 0, \quad s \in S, j \in B
$$

The objective function (1) minimizes the total costs of chartering supply vessels plus locating onshore base(s) to install the central warehouse(s) plus transportation costs of cargoes from suppliers to onshore base(s) and sailing costs from onshore base(s) to operation regions. Constraints (2) and (3) assure that each operation region serves the needed demand and the number of needed weekly visits. Constraints (4) mean that all sending cargoes should not exceed operation region's capacity on a certain day. The limitations of supplier's capacity and onshore base's capacity to send cargoes are indicated by Constraints (5) and (6). Constraints (7) show that all cargoes that enter onshore base(s) from different suppliers must be equal to all cargoes sent to different operation regions in the horizon period. $p$ onshore base(s) is ensured by Constraint (8) exactly. Constraints (9) state that each supply vessel cannot be assigned to more than one onshore base.

Constraints (10) state that the capacity of onshore base(s) is limited by the number of supply vessels. Constraints (11) check the number of days in which each supply vessel can be used during a week; Constraints (12) and (13) show that the duration time of each route should be between $l r$ and $h r$. Constraints (14) mean that a supply vessel does not embark on a new voyage before it returns to the same onshore base(s) after the last voyage. Capacity Constraints (15) show that, for each route on a certain day, the volume of cargoes sent by a supply vessel cannot exceed its capacity. Constraints (16) ensure that each operation region must not be visited more than once on a day. Constraints (17) to (19) mean that, for operation regions requiring two visits, one departure will exist on any three days; for operation regions requiring three visits, at least one departure will exist on any three days; finally, for operation regions requiring four visits, at least two departures will exist on any four days during horizon period. Constraints (20) to (24) define the domains of variables.

\section{Solution methodology}

The methodology presented by Halvorsen-Weare et al. [2] is used to solve the MFSMPLRPTW problem, as depicted in Figure 4, by disregarding the length of voyages and capacity of supply vessels in Phase 1 and by considering the mathematical model. This figure consists of two phases where, in the first phase, all the routes are generated and, in the second phase, the optimal fleet composition, onshore base(s), and the selected routes are determined. The contribution of the voyage generation process is introduced in Section 4.1.

\subsection{Voyage generation}

The voyage generation process is a common method to solve maritime transportation problems. A path flow approach is used instead of an arc flow approach in order to decrease the solution time [2].

The process generates all sailing distances between potential onshore base and a set of operation regions by considering the limitations. Each offshore operation region is not allowed to visit more than once on each voyage. Voyages are supposed to start and finish at the same potential onshore base. There is a pool of supply vessels with different sailing speeds. Therefore, for each subset of operation regions, each onshore base, and each supply vessel, a Traveling Salesman Problem (TSP) is needed to be solved with multiple time windows. In some cases, if the shortest sailing distance is different from the shortest sailing time, the shortest sailing distance will be chosen because of less cost.

It is assumed that the time for loading/unloading cargoes at the potential onshore base is included in the voyage durations. In addition, it is supposed that backloads are considered at the service's time. Ideal weather conditions are supposed and uncontrollable

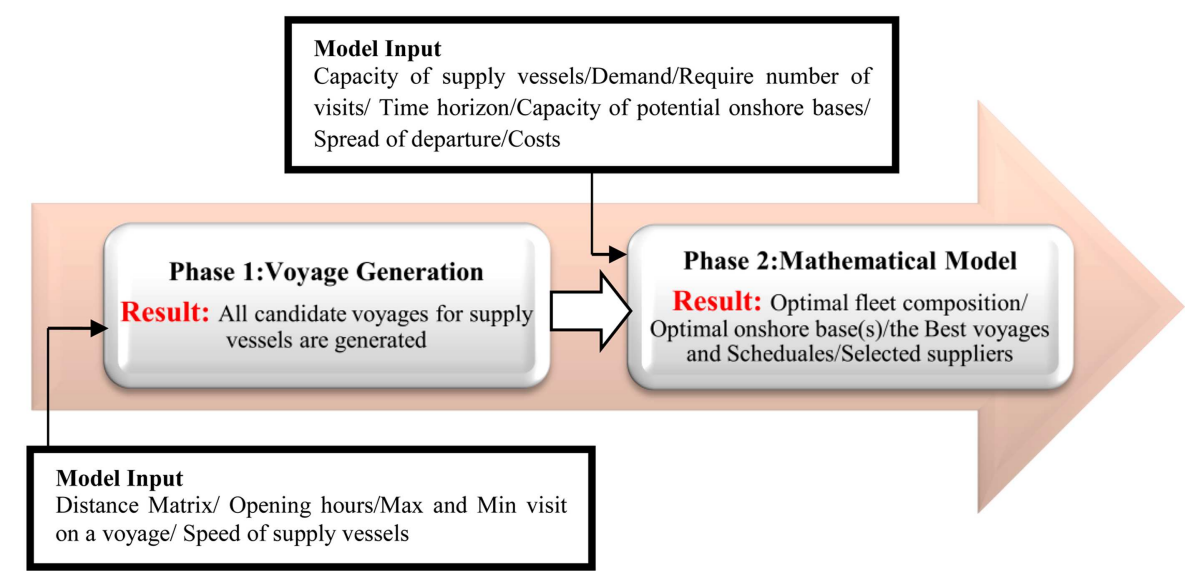

Figure 4. Schematic overview of the methodology. 


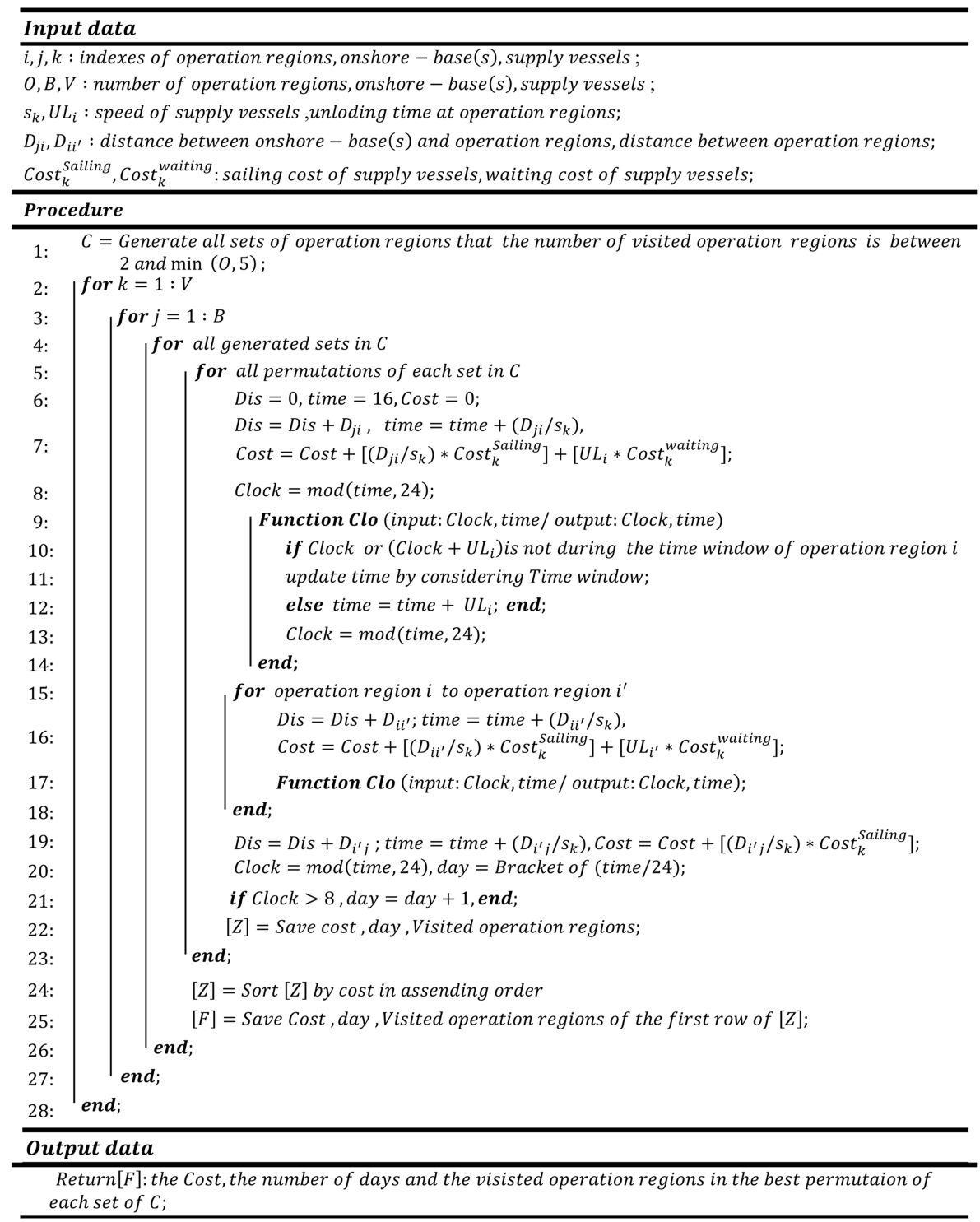

Figure 5. Voyage generation process.

events are not considered. Unlike the previous studies, the capacity of supply vessels and voyage's time are not examined in phase 1 and are considered in the mathematical model. A pseudocode for the new voyage generation process is given in Figure 5 .

\section{Computational results}

The new MFSMPLRPTW model derived from Section 3 has been implemented and tested in real SVP problems faced by the IOOC and by using Matlab software for voyage generator and commercial software GAMS (22.1) for the mathematical model. The aim of the computational study is to test how the model works for a real case in the offshore oil and gas industry, and the results are analyzed in order to discuss the performance of the model. In the following,
Sections 5.1 and 5.2 present the case study and the results, respectively. Section 5.3 shows how the method can be used for the sensitive analysis. Section 5.4 presents a robust schedule for the case study problem.

\subsection{Case description}

The IOOC is one of the world's largest offshore oilproducing companies. The main operation area of the IOOC, as shown in Figure 6, is the Persian Gulf. The IOOC has five offshore operation regions (C1 to $\mathrm{C} 5$ ) and four offices (B1 to B4) along the Persian Gulf and the sea of Oman coastline with private docks to load and unload supply vessels. In addition, there are three purchasing offices (S1 to S3).

Each operation region has a separate set of supply vessels and a separate weekly sailing schedule that is fixed for several months. On the other hand, the 


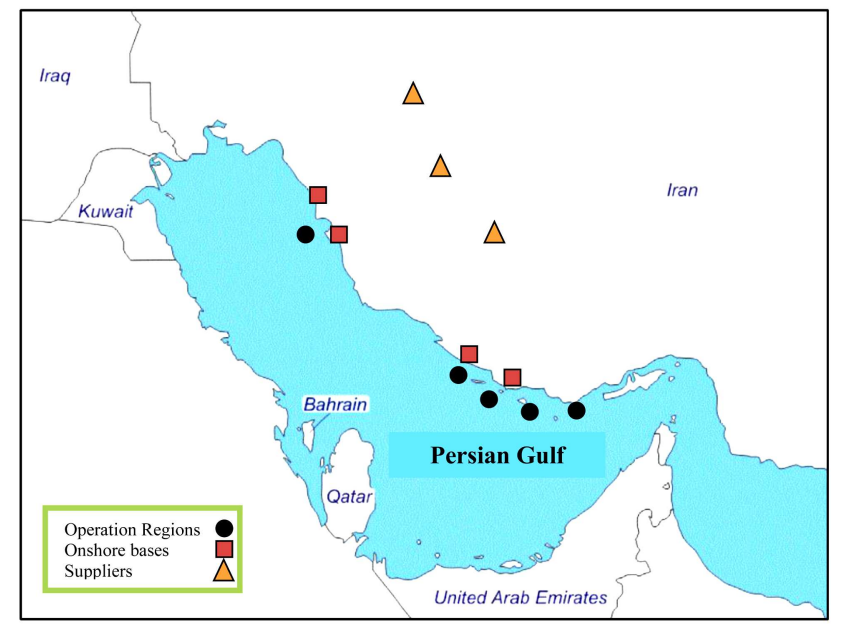

Figure 6. Geographical location of IOOC.

data of operation region's inventories do not merge. In order to solve this problem, the IOOC wants to install central warehouse(s) at one of the potential onshore base. Installing the warehouse(s) without considering transportation costs cannot particularly help the company. Therefore, four potential onshore base(s), at which the IOOC has offices and operational docks, are considered to select the optimal one(s) while keeping the total costs to a minimum.

The total of 37 problem instances, which contain five operational regions, three suppliers, and four potential onshore base(s) have been considered using some real data.

In the case study carried out in the IOOC, the demand of operation regions (by considering a $10 \%$ increase during the next 5 years) varies from 200 to $3000 \mathrm{~m}^{3}$ and the number of weekly required visits is between 2 to 4 times. Operation regions have certain capacities to receive cargoes between 100 and $1000 \mathrm{~m}^{3}$. The service time is between 2 and 7 hours.

The capacity of potential onshore base(s) is considered between 3000 and $8600 \mathrm{~m}^{3}$. In addition, the fixed and variable costs for installing the central warehouse(s) are between 750,000 and $\$ 1,720,000$ and between 1.25 and $\$ 1.375$ for one $\mathrm{m}^{3}$ of cargo, respectively. All onshore bases have opening hours for loading and unloading between 08:00 and 16:00. The number of supply vessels, which could berth in different onshore base(s) and on different days, is between 2 and 6 . All onshore bases will be supplied by 3 suppliers whose capacity varies from 6000 to $16000 \mathrm{~m}^{3}$.
The capacity of supply vessels varies from 1500 to $2300 \mathrm{~m}^{3}$. Time-chartered rates vary from 31500 to $\$ 47250$ for a week. In addition, sailing costs and unloading costs between 100 and $\$ 200$ and between 37.5 and $\$ 50$ for one hour are considered, respectively. The service speed for all supply vessels is different from 8 to 12 knots. The number of hours in a week that supply vessels are available is 144 hours.

All results are obtained by a $2.8 \mathrm{GHz}$, Intel(R) Core(TM) i7 (4CPUs) computer with 8 GB of memory using all available cores. The maximum CPU time for solving GAMS models is set to 5000 seconds.

\subsection{Case study results}

After conducting the MFSMPLRPTW model for the IOOC case, the advantages of this model compared with the current situation appear. Table 2 shows the CPU time for the voyage generation process and mathematical model. The gap shows the optimality gap reported from GAMS software (by using CPLEX solver). In addition, the cost of SVP is shown in the next column. "\#Supply vessel/voyages", refers to the number of supply vessels and voyages, which have been selected to send cargoes. The selected supplier and onshore base(s) are observed in the next columns, respectively.

The cost shown in Table 1 consists of $\$ 1,505,000$ fixed cost to install the central warehouse in Bushehr city and $\$ 476,374.89$ weekly costs. The number of supply vessels can be reduced from four (i.e., current situation) to three, and this corresponds to the total saving of $\$ 37,500$ in a week. Shiraz and Bushehr are selected as the only supplier and onshore-base cities, respectively. By using the MFSMPLRPTW model in the real SVP problem for the IOOC, the acceptable results are obtained.

\subsection{Sensitivity analyses}

Three scenarios in a real case for the IOOC are presented to find whether it is possible to decrease the total cost.

- Scenario 1: Opening some of operation regions during the night.

- Scenario 2: Considering two onshore bases to reduce the risk of rough weather conditions.

- Scenario 3: Reducing the number of weekly visits to operation regions.

Table 2. Optimal results of the MFSMPLRPTW model for the case study.

\begin{tabular}{|c|c|c|c|c|c|c|c|c|}
\hline $\begin{array}{c}\text { Name of } \\
\text { instances } \\
(C-T W-V-P)^{a}\end{array}$ & $\begin{array}{l}\text { CPU time } \\
\text { voyage } \\
\text { generation } \\
\text { (sec.) }\end{array}$ & $\begin{array}{c}\text { CPU time } \\
\text { mathematical } \\
\text { model } \\
\text { (sec.) }\end{array}$ & $\begin{array}{l}\text { Total time } \\
\quad \text { (sec.) }\end{array}$ & $\begin{array}{l}\text { Gap } \\
(\%)\end{array}$ & $\begin{array}{c}\text { Cost } \\
(\$)\end{array}$ & $\begin{array}{l}\text { \#Supply } \\
\text { vessels / } \\
\text { voyages }\end{array}$ & $\begin{array}{l}\text { Selected } \\
\text { supplier }\end{array}$ & $\begin{array}{c}\text { Selected } \\
\text { onshore } \\
\text { base(s) }\end{array}$ \\
\hline $5-5-15-1$ & 5.199 & 215.784 & 220.983 & 0 & $1,981,374.89$ & $3 / 6$ & S3 & B4 \\
\hline
\end{tabular}

a A number of operation regions, which have opening hours, number of needed weekly visits, and number of needed onshore base(s). 
Table 3. Results of Scenario 1.

\begin{tabular}{|c|c|c|c|c|c|c|c|c|c|}
\hline$T W^{a}$ & $\begin{array}{c}\text { CPU time } \\
\text { voyage } \\
\text { (sec.) }\end{array}$ & $\begin{array}{c}\text { CPU time } \\
\text { model } \\
\text { (sec.) }\end{array}$ & $\begin{array}{l}\text { Total } \\
\text { time } \\
\text { (sec.) }\end{array}$ & $\begin{array}{c}\text { Cost } \\
(\$)\end{array}$ & $\begin{array}{l}\text { Selected } \\
\text { vessel } \\
(\mathrm{s})\end{array}$ & $\begin{array}{c}\text { \#Selected } \\
\text { voyage } \\
\text { (s) }\end{array}$ & $\begin{array}{c}\text { Selected } \\
\text { supplier } \\
\text { (s) }\end{array}$ & $\begin{array}{c}\text { Selected } \\
\text { onshore } \\
\text {-base } \\
(\mathrm{s})\end{array}$ & $\begin{array}{c}\text { Savings } \\
\text { for a week } \\
(\$)\end{array}$ \\
\hline $\mathrm{C} 2,3,4,5$ & 7.262 & 80.793 & 88.055 & $1,964,935.988$ & $1,2,3$ & 5 & S3 & B4 & $16,438.902$ \\
\hline $\mathrm{C} 3,4,5$ & 6.627 & 177.60 & 184.23 & $1,964,774.001$ & $1,2,3$ & 5 & S3 & B4 & $16,600.889$ \\
\hline $\mathrm{C} 3,4$ & 5.867 & 275.19 & 281.06 & $1,964,712.642$ & $1,2,3$ & 5 & S3 & B4 & $16,662.248$ \\
\hline $\mathrm{C2}$ & 6.260 & 249.97 & 256.23 & $1,964,712.642$ & $1,2,3$ & 5 & S3 & B4 & $16,662.248$ \\
\hline $\mathrm{C4}$ & 5.737 & 267.35 & 273.09 & $1,964,712.642$ & $1,2,3$ & 5 & S3 & B4 & $16,662.248$ \\
\hline - & 5.345 & 279.04 & 284.39 & $1,964,712.642$ & $1,2,3$ & 5 & S3 & B4 & $16,662.248$ \\
\hline
\end{tabular}

a The operation regions with time windows.

b The amount of savings compared to the case study that comes from IOOC.

Table 4. Results of Scenario 2.

\begin{tabular}{|c|c|c|c|c|c|c|c|}
\hline$C-T W-V-P$ & $\begin{array}{l}\text { CPU time } \\
\text { voyage } \\
\text { generation } \\
\text { (sec.) }\end{array}$ & $\begin{array}{c}\text { CPU time } \\
\text { mathematical } \\
\text { model } \\
\text { (sec.) }\end{array}$ & $\begin{array}{c}\text { Total time } \\
\text { (sec.) }\end{array}$ & $\begin{array}{c}\text { Cost } \\
(\$)\end{array}$ & $\begin{array}{c}\text { \#Supply } \\
\text { vessels/ } \\
\text { voyages }\end{array}$ & $\begin{array}{l}\text { Selected } \\
\text { supplier }\end{array}$ & $\begin{array}{c}\text { Selected } \\
\text { onshore } \\
\text { base(s) }\end{array}$ \\
\hline $5-5-15-2$ & 5.199 & 447.297 & 452.496 & $2,791,317.215$ & $4 / 7$ & S3 & B1 \& B4 \\
\hline \multicolumn{4}{|c|}{ In contrast by $5-5-15-1$} & $+809,942.33$ & $+1 /+1$ & - & $+\mathrm{B} 1$ \\
\hline
\end{tabular}

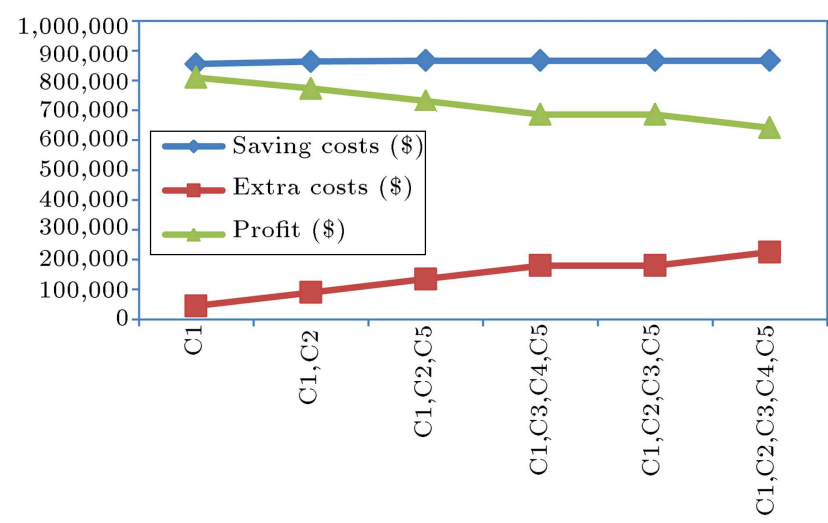

Figure 7. Analysis of not considering TW(s) for operation region(s). $X$ axis shows the operation region(s) without TW(s), and $Y$ axis shows the costs.

For the first scenario, thirty-one problem instances in five groups have been tested by considering all possibilities. The groups have been organized by the number of operation regions that can be open during the nights. In Table 3, the highest savings of each group are shown. It can be seen that, by using Scenario 1, there is no reduction in the number of supply vessels in any group; however, the number of voyages decreases from 6 to 5 .

Analyzing the results shows six alternatives that cost less than the real case study for the IOOC. On the other hand, it has extra costs ( $\$ 3,750$ for a month) if the IOOC wants to have operation regions open during the nights. In Figure 7, the analysis indicates that the best choice for the IOOC is alternative 1. By using this scenario, supply vessels can unload the demand of operation region 1 as soon as they arrive. In this case, the IOOC will save $\$ 809,822.904$ during a year by decreasing the number of voyages from 6 to 5 during the time horizon.

For the second scenario, the results show (Table 4) that the best onshore base(s) for the IOOC are B1 and B4, which are obtained by adding two new constraints to the current model:

- A distance of at least 200 kilometers from each other.

- Sending at least $10 \%$ of total demands to each onshore base.

The result shows that, by this scenario, the IOOC should spend $\$ 809,942.33$ more than the optimal solution. $\$ 750,000$ of this cost results from installing the second warehouse in B1 and $\$ 59,942.33$ of this cost is related to one supply vessel and one voyage more than optimal solution for a week.

In the Persian Gulf and the Sea of Oman, the weather can be rough for six months. If one well stops producing 1,000 barrels per day for five days during six months with a bad weather condition, it does not stand to reason to install the second warehouse because of the cost of extra supply vessels and related voyages, even though sending food and water cannot be postponed regardless of any costs and must be studied more carefully.

In the third scenario, three alternatives obtained by decreasing the total number of weekly visits to 
Table 5. Results of Scenario 3.

\begin{tabular}{|c|c|c|c|c|c|c|c|c|}
\hline Alternative & $\begin{array}{c}\text { Operation } \\
\text { region }\end{array}$ & $\begin{array}{c}\text { CPU } \\
\text { time } \\
\text { voyage } \\
\text { generation } \\
\text { (sec.) } \\
\end{array}$ & $\begin{array}{c}\text { CPU } \\
\text { time } \\
\text { mathematical } \\
\text { model } \\
\text { (sec.) }\end{array}$ & $\begin{array}{c}\text { Total } \\
\text { time } \\
\text { (sec.) }\end{array}$ & $\begin{array}{c}\text { Cost } \\
(\$)\end{array}$ & $\begin{array}{c}\text { \#Supply } \\
\text { vessels/ } \\
\text { voyages }\end{array}$ & $\begin{array}{l}\text { Selected } \\
\text { supplier }\end{array}$ & $\begin{array}{c}\text { Selected } \\
\text { onshore } \\
\text { base(s) }\end{array}$ \\
\hline 1 & $\mathrm{C} 1$ & 5.199 & 151.587 & 156.786 & $1,967,273.61$ & $3 / 6$ & S3 & B4 \\
\hline 2 & $\mathrm{C} 2$ & 5.199 & 140.103 & 145.302 & $1,980,232.97$ & $3 / 6$ & S3 & B4 \\
\hline 3 & C3 & 5.199 & 91.506 & 96.705 & $1,967,467.58$ & $3 / 6$ & S3 & B4 \\
\hline
\end{tabular}

Table 6. Results of robust analysis.

\begin{tabular}{cccccc}
\hline $\begin{array}{c}\text { Problem } \\
\text { instance } \\
(\boldsymbol{C}-\boldsymbol{T} \boldsymbol{W}-\boldsymbol{V})\end{array}$ & $\begin{array}{c}\text { \#Supply } \\
\text { vessels }\end{array}$ & \#Voyages & $\begin{array}{c}\text { \#Onshore } \\
\text { base } \\
(\mathbf{S})\end{array}$ & $\begin{array}{c}\text { Min } \\
\text { slack }^{\mathbf{a}} \\
(\mathbf{m i n})\end{array}$ & $\begin{array}{c}\text { Cost } \\
(\$ \text { for a week })\end{array}$ \\
\hline $5-5-15$ & 3 & 6 & 1 & 217 & $1,981,374.890$ \\
6 hours slack & 4 & 5 & 1 & 548 & $2,010,723.841$ \\
\hline
\end{tabular}

a Minimum slack for all sailed voyages.

operation regions $\mathrm{C} 1, \mathrm{C} 2$, and $\mathrm{C} 3$ from 11 to 10 are tested. The results of the test are shown in Table 5 .

The result shows that the best choice for the IOOC is alternative 1 , which contains operation region $\mathrm{C} 1$ with two weekly visits instead of 3 . By this decision, the IOOC will spend $\$ 14,101.278$ less than the current situation for a week.

The main purpose of SVP is to determine the right number and type of supply vessels. It appears to be impossible to get the optimal solution without considering sailed voyages, supply vessel schedules, and the location of onshore base(s). However, the real scheduling in most cases is different from the plan because of uncontrollable events; therefore, these sensitive analyses can help planners make plans that are more valuable.

\subsection{Robust schedule}

In rough weather, when the height of waves goes above the critical limits, supply vessels cannot sail and have to wait. In some bad weather cases, when it is possible to sail, the speed of supply vessel reduces and the time of voyages increases; moreover, the schedule must be updated. Thus, in order to decrease uncontrollable events like this, a robust scheduling is needed. One way to limit the effects of these events is to add slack time to the voyage time. In Figure 8, the schedule plan of the case study problem is shown. The minimum idle time for all voyages is 217 minutes.

By considering the special weather conditions in the Persian Gulf and the Sea of Oman, adding six hours as a slack time to the voyage time is reasonable (Figure 9 ). By considering this slack time, the minimum idle time increases from 217 minutes to 548 minutes, and

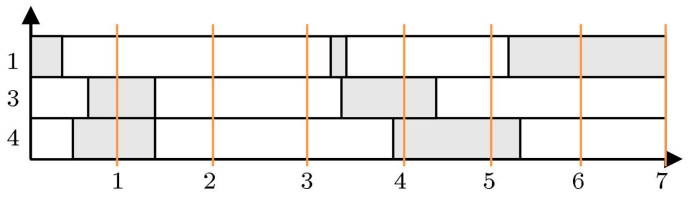

Figure 8. Scheduling plan of the case study problem. $X$ axis shows days of a week, $Y$ axis shows the supply vessels, and gray boxes show the idle time of supply vessels.

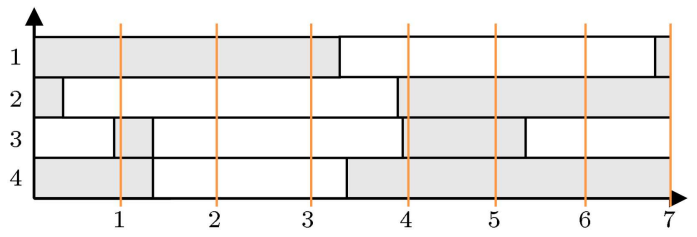

Figure 9. Robust scheduling plan.

the total cost increases about $\$ 30,000$ per week, since one more supply vessel is used. In Table 6 , the detailed results of two options are shown.

\section{Performance of meta-heuristic algorithms}

The experimental results show that the model cannot be solved with GAMS software for medium- and largesized problem instances in a reasonable amount of time. On the other hand, it is proven that swarm-based meta-heuristics are competitive with evolution-based algorithms and physics-based algorithms. Even swarmbased algorithms have some advantages over evolutionbased algorithms [36]. Therefore, the WOA, which is a recent swarm-based meta-heuristic algorithm, was used and its performance in contrast with the PSO algorithm was evaluated in this study. The PSO 
Table 7. Computational results of using meta-heuristic algorithms for small-sized instances.

\begin{tabular}{|c|c|c|c|c|c|c|c|c|c|c|}
\hline \multirow[b]{2}{*}{$\begin{array}{c}\text { Instance } \\
i-j-k-r-s-v\end{array}$} & \multirow[b]{2}{*}{ \#Variables } & \multicolumn{3}{|c|}{ PSO } & \multicolumn{3}{|c|}{ WOA } & \multicolumn{3}{|c|}{ GAMS (CPLEX solver) } \\
\hline & & $\begin{array}{c}\text { Mean } \\
\text { gap/ } \\
100 \\
(\%)\end{array}$ & $\begin{array}{c}\text { \#Voyages- } \\
\text { \#supply } \\
\text { vessels }\end{array}$ & $\begin{array}{c}\text { \#Onshore } \\
\text { bases- } \\
\text { \#suppliers }\end{array}$ & $\begin{array}{c}\text { Mean } \\
\text { gap/ } \\
100 \\
(\%)\end{array}$ & $\begin{array}{c}\text { \# Voyages- } \\
\text { \#supply } \\
\text { vessels }\end{array}$ & $\begin{array}{c}\text { \#Onshore } \\
\text { bases- } \\
\text { \#suppliers }\end{array}$ & $\begin{array}{l}\text { Total } \\
\text { time } \\
\text { (sec.) }\end{array}$ & $\begin{array}{c}\text { \#Voyages- } \\
\text { \#supply } \\
\text { vessels }\end{array}$ & $\begin{array}{c}\text { \#Onshore } \\
\text { bases- } \\
\text { \#suppliers }\end{array}$ \\
\hline $3-2-5-4-2-9$ & 1,136 & 0.026 & $6-3$ & $1-2$ & 0.026 & $6-3$ & $1-2$ & 1.99 & $5-2$ & $1-2$ \\
\hline $3-2-5-4-3-10$ & 1,138 & 0.039 & $6-3$ & $1-1$ & 0.039 & $6-3$ & $1-1$ & 2.94 & $5-2$ & $1-1$ \\
\hline $3-3-5-4-3-10$ & 1,707 & 0.040 & $6-4$ & $1-1$ & 0.040 & $6-4$ & $1-1$ & 8.33 & $5-3$ & $1-1$ \\
\hline $3-4-5-4-3-10$ & 2,276 & 0.025 & $6-3$ & $1-1$ & 0.025 & $6-3$ & $1-1$ & 18.07 & $5-3$ & $1-1$ \\
\hline $3-4-5-4-4-11$ & 2,280 & 0.044 & $7-4$ & $1-1$ & 0.045 & $7-4$ & $1-1$ & 18.82 & $5-3$ & $1-1$ \\
\hline $4-2-5-11-2-11$ & 3,866 & 0.042 & $6-3$ & $1-1$ & 0.042 & $6-3$ & $1-1$ & 25.70 & $5-2$ & $1-1$ \\
\hline $4-2-5-11-3-12$ & 3,868 & 0.024 & $6-3$ & $1-1$ & 0.024 & $6-3$ & $1-1$ & 45.97 & $5-3$ & $1-1$ \\
\hline $4-3-5-11-3-12$ & 5,802 & 0.024 & $6-3$ & $1-1$ & 0.024 & $6-3$ & $1-1$ & 54.41 & $5-3$ & $1-1$ \\
\hline $4-4-5-11-4-13$ & 7,740 & 0.042 & $6-4$ & $1-1$ & 0.063 & $6-4$ & $1-1$ & 62.16 & $5-3$ & $1-1$ \\
\hline $5-4-5-26-3-15$ & 21,876 & 0.055 & $7-5$ & $1-1$ & 0.057 & $7-5$ & $1-1$ & 1347.16 & $6-3$ & $1-1$ \\
\hline
\end{tabular}

Table 8. Computational results of using the meta-heuristics for medium- and large-sized instances.

\begin{tabular}{|c|c|c|c|c|c|}
\hline \multirow{2}{*}{$\begin{array}{c}\text { Instance } \\
i-j-k-r-s-v\end{array}$} & \multirow{2}{*}{ \#Variables } & \multirow{2}{*}{$\begin{array}{c}\text { PSO } \\
\text { Obj. value }\end{array}$} & \multirow{2}{*}{$\begin{array}{c}\text { WOA } \\
\text { Obj. value }\end{array}$} & \multicolumn{2}{|c|}{ GAMS } \\
\hline & & & & Obj. value ${ }^{a}$ & Gap (\%) \\
\hline $6-4-10-56-3-16$ & 109,816 & $2,890,052$ & $2,936,113$ & $2,774,509$ & 2.33 \\
\hline $7-4-10-112-3-17$ & 250,936 & $2,972,781$ & $2,915,638$ & $2,829,943$ & 3.36 \\
\hline 8-4-10-210-3-19 & 529,256 & $3,050,654$ & $3,116,021$ & $3,515,883$ & 31.84 \\
\hline $9-4-10-372-3-21$ & $1,041,656$ & $3,072,100$ & $3,144,600$ & $3,722,216$ & 35.13 \\
\hline $10-4-10-627-3-13$ & $1,931,216$ & $3,267,160$ & 3,375198 & NA & \\
\hline
\end{tabular}

a The objective function value obtained by solving different problem instances and different methods.

algorithm is one of the most popular swarm-based algorithms. Furthermore, Mirjalili and Lewis [36], who has recently proposed the WOA, has compared their algorithm with the PSO to evaluate its performance.

Similar to most meta-heuristic algorithms, PSO and WOA have several parameters that need to be tuned before reaching appropriate results. The Taguchi method in Minitab software is used to reduce the variance of the experiments, which is based on the Design Of Experiments (DOE) with optimization of control parameters to obtain the best results [46].

In order to evaluate the performance of using meta-heuristic algorithms, the results of randomly generated small-sized instances are compared (see Table 7) with the optimal solutions obtained with GAMS software by using the CPLEX solver. When the number of the problem variables rises more than 100 thousand, GAMS software cannot find the optimal solution during 5,000 seconds. Based on Table 8, the results show that PSO and WOA have a considerable performance to solve medium- and large-sized problems. The results of medium instances obtained by GAMS software are better than the forgoing metaheuristics, because the gap value is about 2 and $3 \%$, implying that the obtained objective values are optimal roughly.
Meta-heuristic algorithms are tested on a set of 10 small examples, whose data are provided by the IOOC. The size of problems (i.e., number of variables) varies from 1136 to 21876 , and each instance is run 40 times by every algorithm. Since the data of real mediumand large-sized instances do not exist, in order to show how our algorithms can be useful for real mediumand large-sized cases, five medium- and large-sized instances are generated randomly.

The name of problem instances consists of six numbers separated by a hyphen (i.e., $i-j-k-r-s-v$ ). The first number indicates the number of operation regions; the second one shows the number of potential onshore base(s); the third one represents the number of supply vessels. The fourth and fifth ones show the number of routes and suppliers, respectively, and the sixth one shows the number of weekly visits to all operation regions. The column "Mean gap (\%)" gives the percentage of the deviation of any algorithm (WOA or PSO). The evaluation of the gap between a metaheuristic algorithm and the exact method for smallsized instances is computed as:

$$
\operatorname{Gap}_{a}=\left(\frac{\left[Z_{a}-Z_{\text {best }}\right]}{Z_{\text {best }}}\right) * 100
$$

where $Z_{a}$ is the fitness of the algorithm, and $Z_{b e s t}$ is the best solution ever found for a random instance. 
Table 9. Results of ANOVA test for instances.

\begin{tabular}{cccccccc}
\hline & & DF & SS & MS & $\boldsymbol{F}$-value & $\boldsymbol{P}$-value & Test results \\
\hline \multirow{2}{*}{ Small } & PSO-WOA & 1 & 0.29 & 0.29 & 0.19 & 0.672 & Means are equal \\
instances & Error & 18 & 27.89 & 1.55 & & & \\
\hline Medium and & Total & 19 & 28.18 & & & & \\
PSO-WOA & 1 & 2.10 & 2.10 & 1.09 & 0.337 & \multirow{2}{*}{ Means are equal } \\
instances & Error & 6 & 11.61 & 1.93 & & & \\
\hline
\end{tabular}

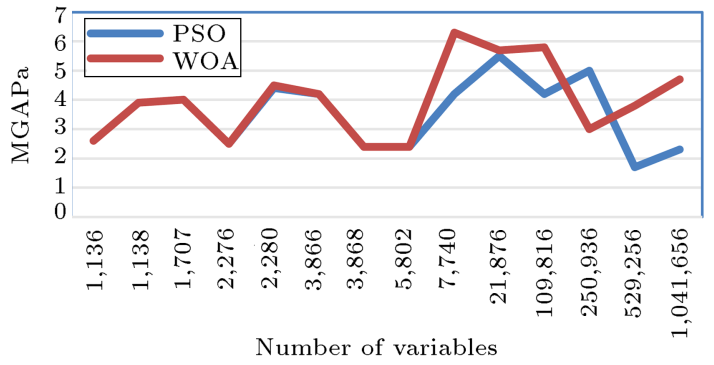

Figure 10. "MGAP" comparison of WOA with PSO.

For the small-sized instances, $Z_{\text {best }}$ is the optimal solution obtained by GAMS software (CPLEX solver). For the medium- and large-sized problems, it is the best solution ever found by meta-heuristic algorithms or GAMS solver. Since each experiment includes 40 observations, we have used the mean value of the $\operatorname{GAP}\left(M G A P_{a}\right)$ to address the performances of the meta-heuristics.

$$
M G A P_{a}=\frac{1}{40} \sum_{i=1}^{40} \operatorname{Gap}_{a_{i}}
$$

where $\operatorname{Gap}_{a_{i}}$ is the percentage of deviation for observation $i$ of the algorithm.

The graphical analysis of the results (Figure 10) states that the performance of the WOA is the same as or better than PSO for $71.4 \%$ of the experiments.

The statistical analysis presented in Table 9 indicates similar competence for WOA and PSO algorithms to solve this kind of problem. Since the performance of PSO algorithm for LRPs is proven, we can state that WOA is a suitable algorithm for this kind of optimization problems.

\section{Conclusion}

In this paper, a new real model for a Maritime Fleet Sizing Mix Periodic Location-Routing Problem with Time Windows (MFSMPLRPTW) has been studied. A two-stage solution approach was used, where all candidate routes were generated in the first phase, and the optimal fleet composition, onshore base(s), and voyages were determined in the second phase. According to the IOOC, the annual cost savings of installing a central warehouse (i.e., one onshore base) and using three supply vessels instead of four (i.e., current situation) would be about $\$ 2$ million. Additionally, the sensitivity analyses showed that if only operation region 1 could be open during the nights, the annual cost savings would be about $\$ 855,000$. By locating two onshore bases in order to have a reliable schedule in a rough weather condition, the total cost would increase about $\$ 809,000$, which seemed not logical. In another analysis, the results indicated that, by decreasing the number of weekly visits from 3 to 2 for operation region 1 , the annual saving costs would be about $\$ 730,000$. Robust scheduling showed that, by spending about $\$ 30,000$ for a week, the IOOC could be sure that the schedules would not change during several months. The computational study indicated that the small-sized problems could be solved by exact methods using GAMS software (CPLEX solver) in a reasonable amount of time; however, the medium- and large-sized instances could not be solved. Therefore, two metaheuristic algorithms (i.e., PSO and WOA) were used for large-sized instances. By making a balance between exploration and exploitation perfectly, escaping local minima, and also using a logarithmic spiral function to search the border area, The WOA, which is a recent swarm-based meta-heuristic algorithm, showed an acceptable performance to solve LRPs. The graphical and statistical analyses indicated similar competence for the WOA and PSO algorithms to solve this kind of problem.

In this paper, a simple way to reduce the risk of increasing the time of voyages was studied. Therefore, the robustness approach can be presented in the future by considering more uncontrollable events. On the other hand, the environmental aspect of supply vessels and their voyages is another important aspect, which can be discussed for the future study by using multiobjective models. Furthermore, using an exact method (e.g., column generation schemes) in order to solve large-sized problem instances can be noticed.

\section{Acknowledgements}

This research is funded and carried out as a part of the Technology and Research Department of the Iranian Offshore Oil Company (IOOC) as a member 
of the National Iranian Oil Company (NIOC) and is gratefully acknowledged. We would also like to express our gratitude to the reviewers for their valuable comments.

\section{References}

1. Cuesta, E.F., Andersson, H., Fagerholt, K., and Laporte, G. "Vessel routing with pickups and deliveries: an application to the supply of offshore oil platforms", Computer and Operations Research, 79, pp. 140-147 (2017).

2. Halvorsen-Weare, E.E., Fagerholt, K., Nonås, L.M., and Asbjørnslett, B.E. "Optimal fleet composition and periodic routing of offshore supply vessels", European Journal of Operational Research, 223(2), pp. 508-517 (2012).

3. Pantuso, G., Fagerholt, K., and Hvattum, L.M. "A survey on maritime fleet size and mix problems", European Journal of Operational Research, 235(2), pp. 341-349 (2014).

4. Christiansen, M., Fageholt, K., Nygreen, B., and Ronen, D. "Maritme transportation", Transportation, 14, pp. 189-284 (2006).

5. Everett, J.I., Hax, A.C., Lewinson, V.A., and Nudds, D. "Optimization of a fleet of large tankers and bulkers: a linear programming approach", Marine Technology, 9(4), pp. 430-438 (1972).

6. Murotsu, Y. and Taguchi, K. "optimization of ship fleet-size", Bulletin of University of Osaka Prefecture, Series A: Engineering and Natural Science, 23, pp. 171-192 (1975).

7. Larson, R.C. "Transporting sludge to the 106-mile site: an inventory/routing model for fleet sizing and logistics system design", Transportation Science, 22, pp. 186198 (1988).

8. Pesenti, R., "Hierarchical resource planning for shipping companies", European Journal of Operational Research, 86(1), pp. 91-102 (1995).

9. Sigurd, M.M., Ulstein, N.L., Nygreen, B., and Ryan, D.M., "Ship scheduling with recurring visits and visit separation requirements", In Column Generation, Springer, pp. 225-245 (2005).

10. Zeng, Q. and Yang, Z. "Model integrating fleet design and ship routing problems for coal shipping", Lecture Notes in Computer Science, 4489, pp. 1000-1003 (2007).

11. Gogna, A. and Tayal, A. "Meta-heuristics: review and application", Journal of Experimental \& Theoretical Artificial Intelligence, 25(4), pp. 503-526 (2013).

12. Fagerholt, K., Christiansen, M., Hvattum, L.M., Johnsen, T.A.V., and Vab, T.J. "A decision support methodology for strategic planning in maritime transportation", Omega, 38(6), pp. 465-474 (2010).

13. Dantzig, G. and Ramser, J. "The truck dispatching problem", Management Science, 6(1), pp. 80-91 (1959).
14. Kocü, C., Bektasü, T., Jabali, O., and Laporte, G. "The fleet size and mix location-routing problem with time windows: formulations and a heuristic algorithm", European Journal of Operation Research, 248 (1), pp. 33-51 (2016).

15. Fagerholt, K. and Lindstad, H. "Optimal policies for maintaining a supply service in the Norwegian Sea", Omega, 28(3), pp. 269-275 (2000).

16. Aas, B., Gribkovskaia, I., Halskau, Ø., and Shlopak, A. "Routing of supply vessels to petroleum installations", International Journal of Physical Distribution \& Logistics Management, 37(2), pp. 164-179 (2007).

17. Gribkovskaia, I., Laporte, G., and Shlopak, A. "A tabu search heuristic for a routing problem arising in servicing of offshore oil and gas platforms", Journal of the Operational Research Society, 59(11), pp. 14491459 (2007).

18. Iachan, R. "A Brazilian experience: 40 years using operations research at Petrobras", International Transactions in Operational Research, 16(5), pp. 585593 (2009).

19. Aas, B., Halskau Sr, Ø., and Wallace, S.W. "The role of supply vessels in offshore logistics", Maritime Economics and Logistics, 11(3), pp. 302-325 (2009).

20. Shyshou, A., Gribkovskaia, I., and Barceló, J. "A simulation study of the fleet sizing problem arising in offshore anchor handling operations", European Journal of Operational Research, 203(1), pp. 230-240 (2010).

21. Halvorsen-Weare, E.E. and Fagerholt, K. "Robust supply vessel planning", Network Optimization, 6701, pp. 559- 573 (2011).

22. Shyshou, A., Gribkovskaia, I., Laporte, G., and Fagerholt, K. "A large neighbourhood search heuristic for a periodic supply vessel planning problem arising in offshore oil and gas operations", Information Systems and Operational Research, 50(4), pp. 195-204 (2012).

23. Norlund, E.K., Gribkovskaia, I., and Laporte, G. "Supply vessel planning under cost, environment and robustness considerations", Omega, 57(B), pp. 271-281 (2015).

24. Christiansen, M., Fagerholt, K., Rachaniotis, N., and Stålhane, M. "Operational planning of routes and schedules for a fleet of fuel supply vessels", Transportation Research Part E: Logistics and Transportation Review, 105, pp. 163-175 (2017).

25. Prodhon, C. and Prins, C. "A survey of recent research on location-routing problems", European Journal of Operational Research, 238(1), pp. 1-17 (2014).

26. Nagy, G. and Salhi, S. "Location-routing: Issues, models and methods", European Journal of Operational Research, 177(2), pp. 649-672 (2007). 
27. Prins, C., Prodhon, C., and Wolfler-Calvo, R. "Solving the capacitated location routing problem by a GRASP complemented by a learning process and a path relinking”, 4OR: A Quarterly Journal of Operations Research, 4(3), pp. 221-238 (2006).

28. Derbel, H., Jarboui, B., Hanafi, S., and Chabchoub, H. "Genetic algorithm with iterated local search for solving a location-routing problem", Expert Systems with Applications, 39(3), pp. 2865-2871 (2012).

29. Yu, V.F., Lin, S.W., Lee, W., and Ting, C.J. "A simulated annealing heuristic for the capacitated location routing problem", Computers and Industrial Engineering, 58(2), pp. 288-299 (2010).

30. Hemmelmayr, V.C., Cordeau, J.-F., and Crainic, T.G. "An adaptive large neighborhood search heuristic for two-echelon vehicle routing problems arising in city logistics", Computers and Operations Research, 39(12), pp. 3215-3228 (2012).

31. Jarboui, B., Derbel, H., Hanafi, S., and Mladenovic, N. "Variable neighborhood search for location routing", Computers and Operations Research, 40(1), pp. 47-57 (2013).

32. Laporte, G. and Nobert, Y. "An exact algorithm for minimizing routing and operating costs in depot location", European Journal of Operational Research, 6(2), pp. 224-226 (1981).

33. Albareda-Sambola, M., Diaz, J.A., and Fernandez, E. "A compact model and tight bounds for a combined location-routing problem", Computers and Operations Research, 32(3), pp. 407-428 (2005).

34. Belenguer, J.M., Benavent, E., Prins, C., Prodhon, C., and Wolfler-Calvo, R. "A branch-and-cut method for the capacitated location-routing problem", Computers and Operations Research, 38(6), pp. 931-941 (2011).

35. Kennedy, J. and Eberhart, R. "Particle swarm optimization", Proceedings of the 1995 IEEE International Conference on Neural Networks, pp. 1942-1948 (1995).

36. Mirjalili, S., Lewis, A. "The whale optimazation algorithm", Advances in Engineering Software, 95, pp. 51-67 (2016).

37. Marinakis, Y. and Marinaki, M. "A particle swarm optimization algorithm with path relinking for the location routing problem", Journal of Mathematical Modeling and Algorithms, 7(1), pp. 59-78 (2008).

38. Belmecheri, F., Prins, C. Yalaoui, F., and Amodeo, L. "A particle swarm optimization algorithm for a vehicle routing problem with heterogeneous fleet, mixed backhauls, and time windows", Journal of Intelligent Manufacturing, 24(4), pp. 775-789 (2013).

39. Onwunalu, J.E. and Durlofsky, L.J. "Application of a particle swarm optimization algorithm for determining optimum well location and type", Computational Geosciences, 14(1), pp. 183-198 (2010).
40. Kaveh, A. and Ghazaan, M.I "Enhanced whale optimization algorithm for sizing optimization of skeletal structures", Mechanics Based Design of Structures and Machines, 45(3), pp. 345-362 (2016).

41. Mafarja, M.M. and Mirjalili, S. "Hybrid whale optimization algorithm with simulated annealing for feature selection", Neurocomputing, 260, pp. 302-312 (2017).

42. Prakash, D.B. and Lakshminarayana, C. "Optimal siting of capacitors in radial distribution network using whale optimization algorithm", Alexandria Engineering Journal, 56(4), pp. 499-509 (2017).

43. Reddy, P.D.P., Reddy, V.C.V., and Manohar, T.G. "Whale optimization algorithm for optimal sizing of renewable resources for loss reduction in distribution systems", Renewables: Wind, Water and Solar, 4(1), 3 (2017).

44. Abd El Aziz, M., Ewees, A.A., and Ella Hassanein, A. "Whale optimization algorithm and moth-flame optimization for multilevel thresholding image segmentation", Expert Systems With Applications, 83(C), pp. 242-256 (2017).

45. Fruggiero, F., Lambiase, A., Macchiaroli, R., and Miranda, S. "The role of uncertainty in supply chains under dynamic modeling", International Journal of Industrial Engineering Computations, 8(1), pp. 119140 (2017).

46. Rincon-Garcia, N., Waterson, B.J., and Cherrett, T.J. "A hybrid metaheuristic for the time-dependent vehicle routing problem with hard time windows", International Journal of Industrial Engineering Computations, 8(1), pp. 141-160 (2017).

\section{Biographies}

Mohsen Amiri is currently a PhD student under Professor Seyed Jafar Sadjadi's supervision at Islamic Azad University-Science and Research Branch in Iran. He obtained his MSc in Industrial Engineering from Isfahan University of Technology in Iran (2006) and his BSc in Industrial Engineering from Islamic Azad University, North Tehran Branch, in Iran (2003). His research interests include supply chain, mathematical modeling, and location-routing problems.

Seyed Jafar Sadjadi is a Professor of Industrial Engineering at Iran University of Science and Technology in Iran. He received his $\mathrm{PhD}$ degree at University of Waterloo in Canada. His research interests include solving different classes of optimization problems in industrial engineering areas (e.g., supply chain management, portfolio optimization, and optimal pricing). He has been serving at Iran University of Science and Technology since 2001.

Reza Tavakkoli-Moghaddam is a Professor of Industrial Engineering at the College of Engineering, 
University of Tehran in Iran. He obtained his PhD in Industrial Engineering from Swinburne University of Technology in Melbourne (1998). He serves as a member of Editorial Board in five reputable academic journals. He is the recipient of the 2009 and 2011 Distinguished Researcher Awards and the 2010 and 2014 Distinguished Applied Research Awards at University of Tehran, Iran. He has been selected as the National Iranian Distinguished Researcher in 2008 and 2010 by the Ministry of Science, Research, and Technology (MSRT) in Iran. He obtained the outstanding rank as the top $1 \%$ scientist and researcher in the world elite group, reported by Thomson Reuters in 2014. He has published 4 books, 17 book chapters, and more than 700 journal, and conference papers.

Armin Jabbarzadeh is an Assistant Professor at the Department of Industrial Engineering at Iran University of Science and Technology. He received his $\mathrm{PhD}$ in Industrial Engineering from Iran University of Science and Technology. He has been involved in a variety of research projects about supply chain and logistics optimization. His research interests include supply chain network design, production planning, and applied operations research under uncertainty. 